EPJ manuscript No.

(will be inserted by the editor)

\title{
Wigner-localized states in spin-orbit-coupled bosonic ultracold atoms with dipolar interaction
}

\author{
Y. Yousefii ${ }^{1}$, E. Ö. Karabulut ${ }^{1,3}$, F. Malet ${ }^{1,2}$, J. Cremon ${ }^{1}$, and S. M. Reimann ${ }^{1}$ \\ 1 Mathematical Physics, Lund University, LTH, P.O. Box 118, SE-22100 Lund, Sweden \\ 2 Department of Theoretical Chemistry and Amsterdam Center for Multiscale Modeling, \\ FEW, Vrije Universiteit, De Boelelaan 1083, 1081HV Amsterdam, The Netherlands \\ 3 Department of Physics, Faculty of Science, Selcuk University, TR-42075, Konya, Turkey
}

\begin{abstract}
We investigate the occurence of Wigner-localization phenomena in bosonic dipolar ultracold few-body systems with Rashbalike spin-orbit coupling. We show that the latter strongly enhances the effects of the dipole-dipole interactions, allowing to reach the Wignerlocalized regime for strengths of the dipole moment much smaller than those necessary in the spin-orbit-free case.
\end{abstract}

\section{Introduction}

During the last recent years, one of the most active topics in the field of ultracold atom gases has been the study of spin-orbit ( $\mathrm{SO}$ ) coupling effects, whose realization was discovered to be possible by means of properly designed atom-laser coupling schemes [1]. The physics of such SO-coupled ultracold gases is described by a Hamiltonian rather similar to the one well known from semiconductor-nanostructure systems, where so-called Rashba and Dresselhaus SO effects arise naturally due to the presence of internal net electric fields [2]. Already the first theoretical studies on SO coupling in ultracold atomic gases [1] showed the innovative potential of these systems, opening the door for the investigation of a collection of new physical phenomena with no analogue in their solid-state counterparts [134. Research in this field became even more intense with the recent first experimental realization of a SO-coupled Bose-Einstein condensate 5 .

The interplay between the SO coupling and the atom-atom interactions is known to give rise to novel physical properties [3] in both the strongly-correlated [6] and in the weakly-interacting regimes [4. Indeed, it has been shown that the SO coupling can enhance interaction effects 78 , and this issue is at the focus of the present article. In particular, we consider a symmetric Rashba-type SO coupling [9, which is the most frequently used form in the literature due to its similarity to that known from semiconductor nanostructures. However, it has not yet been experimentally realized due to its highly symmetric structure.

Wigner-localization properties of quasi-one- and quasi-two-dimensional dipolar systems have been previously addressed in the absence of SO coupling [1011]. Here, we investigate how the presence of SO coupling enhances the Wigner-localization effects in few-body dipolar ultracold bosonic systems, finding a clear analogy with what 
was previously observed in semiconductor quantum dots [12 with genuine Rashba coupling and electron-electron Coulomb interactions [13.

The paper is organized as follows. In Sec. 2 we introduce the model and briefly describe the theoretical approach. In Sec. 3 we present our results, and in Sec. 4 we draw conclusions and give an outlook.

\section{Model and method}

We consider a few ultracold bosonic atoms with mass $m$ confined in the $x y$-plane by a harmonic potential with a strong confinement along the $z$ direction, i.e., $V_{\text {trap }}(x, y, z)=$ $m\left(\omega_{0}^{2}\left(x^{2}+y^{2}\right)+\omega_{z}^{2} z^{2}\right) / 2$ with trap frequencies satisfying $\omega_{z} \gg \omega_{0}$. This effectively freezes the motion of atoms in the $z$ direction, yielding a quasi-two-dimensional system. In what follows, we express the lengths in units of $l_{0}$, where $l_{0}=\sqrt{\hbar /\left(m \omega_{0}\right)}$ is the oscillator length in the $x y$-plane, and energies in units of $\hbar \omega_{0}$.

The atoms have two internal (pseudo-spin) states that are coupled with a properly designed Raman laser scheme, giving rise to SO coupling [5]. Although the form of the coupling, which has been realized so far by the current experiments [35], is not purely symmetric, we assume here a symmetric Rashba-type SO coupling because of being the most commonly addressed form in the literature 9] and its similarity to that in semiconductor nanostructures [13. The single-particle Hamiltonian of the system then reads

$$
H_{\mathrm{sp}}=\left(\frac{1}{2} \mathbf{p}^{2}+\frac{1}{2} \mathbf{r}^{2}\right)+k_{\mathrm{SO}}\left(\hat{\sigma_{x}} \mathbf{p}_{y}-\hat{\sigma_{y}} \mathbf{p}_{x}\right)
$$

where $\mathbf{p}=\left(p_{x}, p_{y}\right)$ is the momentum operator, $\mathbf{r}=(x, y)$ is the position vector in the $x y$-plane, $k_{\mathrm{SO}}$ determines the strength of the SO coupling and the $\hat{\sigma}$ 's are the $2 \times 2$ Pauli spin matrices.

Regarding the two-body interactions, we assume that each atom has a dipole moment $\mathbf{d}$ and they are oriented, by means of an external field, along the same direction in the $x z$-plane forming an angle $\Theta$ with respect to the $x$-axis. In the particular case $\Theta=90^{\circ}$, the dipoles are aligned perpendicularly to their plane of motion, and the dipole-dipole interaction (DDI) is isotropic and purely repulsive [14151617. As the dipoles are tilted (i.e., $\Theta$ is decreased) the DDI becomes anisotropic with increasing attractive regions. Accordingly, the many-body Hamiltonian reads

$$
\mathcal{H}=\sum_{i=1}^{N}\left(H_{\mathrm{sp}}\right)_{i}+\frac{1}{2} \sum_{i \neq j}^{N} V_{\mathrm{dip}}^{2 \mathrm{D}}\left(\mathbf{r}_{\mathbf{i}}-\mathbf{r}_{\mathbf{j}}\right)
$$

where the effective dipolar interaction $\left(V_{\text {dip }}^{2 \mathrm{D}}\right)$ in the $x y$-plane is given by $[16]$

$$
\begin{array}{r}
\frac{V_{\mathrm{dip}}^{2 \mathrm{D}}(\mathbf{r})}{\hbar \omega_{0}}=\frac{D^{2}}{\sqrt{8 \pi}} \frac{e^{\tilde{r}_{\perp}^{2}}}{l_{z}^{3}}\left\{\left(2+4 \tilde{r}_{\perp}^{2}\right) K_{0}\left(\tilde{r}_{\perp}^{2}\right)-4 \tilde{r}_{\perp}^{2} K_{1}\left(\tilde{r}_{\perp}^{2}\right)\right. \\
+\cos ^{2} \Theta\left[-\left(3+4 \tilde{r}_{\perp}^{2}\right) K_{0}\left(\tilde{r}_{\perp}^{2}\right)+\left(1+4 \tilde{r}_{\perp}^{2}\right) K_{1}\left(\tilde{r}_{\perp}^{2}\right)\right] \\
\left.+2 \cos ^{2} \Theta \cos ^{2} \phi\left[-2 \tilde{r}_{\perp}^{2} K_{0}\left(\tilde{r}_{\perp}^{2}\right)+\left(2 \tilde{r}_{\perp}^{2}-1\right) K_{1}\left(\tilde{r}_{\perp}^{2}\right)\right]\right\},
\end{array}
$$

with $l_{z}=\sqrt{\hbar / m \omega_{z}}$ being the oscillator length in the $z$-direction, and $\tilde{r}_{\perp} \equiv r /\left(2 l_{z}\right)$. Here, $\phi$ is the angle in cylindrical polar coordinates, $K_{0}$ and $K_{1}$ are the zeroth- and 
first-order modified Bessel functions of the second kind. The coupling constant $D$ is defined in terms of the dipole moment of the atoms as $D=\frac{d}{\sqrt{4 \pi \epsilon_{0}}} \frac{\sqrt{m}}{\hbar \sqrt{l_{0}}}[16]$.

Since in this work we focus on Wigner-localization effects, we only consider the case with $54.7^{\circ}<\Theta \leq 90^{\circ}$ for which the three-dimensional form of the interaction is repulsive [16].

We numerically obtain the eigenstates of the many-body Hamiltonian Eq. (2) by means of a configuration-interaction (CI) approach [16. For the problem at hand, instead of the usual choice of 2D harmonic oscillator orbitals as basis for expanding the many-body states, it is more convenient to construct a new basis by including as well the SO term of the single-particle Hamiltonian. This allows for a more natural description of the SO coupling effects in the system and to perform the numerical simulations in a more efficient way. As usually done in CI calculations, we truncate the number of basis states to a finite but large enough subset that provides a converged solution. Due to the rapid growth of the basis size with the number of particles, we restrict our simulations to $N \leq 4$.

\section{Results}

We first study the case where the dipoles are oriented perpendicularly to the $x y$-plane $\left(\Theta=90^{\circ}\right)$. Figs. 1 and 2 show the densities and pair-correlated densities obtained for a system with, respectively, three and four dipoles considering different values of the Rashba SO coupling $\lambda_{\mathrm{SO}}$ (dimensionless parameter defined by $\lambda_{\mathrm{SO}}=l_{0} k_{\mathrm{SO}}$ ) and for a fixed strength of the dipolar interaction $D$. The chosen value for the latter corresponds, in the SO-free case, to a moderate interaction strength and to a system in the "liquid state", with a rather homogeneous density and a pair-correlation density [16] showing no signs of Wigner localization. One can see from both figures that this is indeed the case when the SO coupling is small (here $\lambda_{\mathrm{SO}}=0.4$ ): the SO term acts only as a small perturbation and the obtained results are qualitatively very similar to those corresponding to the case without SO coupling [16.

As the strength of the SO coupling is increased, however, the densities (Figs. 10 and $2 \mathrm{~b}$ ) start to develop a sharper and more localized structure, forming a ringshaped profile that reveals a strong mutual repulsion. This tendency can also be seen from the corresponding pair-correlation densities (Figs. 1 $\mathrm{d}$ and 2 $\mathrm{d}$ ), which show characteristic Wigner-localized structures (triangular and rectangular for $N=3$ and 4 , respectively), with a number of clearly marked peaks equal to $N-1$ in each case. In the absence of SO coupling, such Wigner-localized configurations are only observed for much stronger dipolar interaction strengths (for example, in the case of $N=3$ dipolar bosons similar localized states are found for $D=5[16$, and here it should be stressed that the interaction strength increases as $D^{2}$ ). Our results thus clearly show that the presence of SO coupling substantially enhances the effect of dipolar interaction in the system, analogously as the effect previously observed in Rashba-coupled semiconductor quantum dots with Coulomb interaction 13 . We have also found that this effect remains in the case of tilted dipoles when the interaction becomes anisotropic. Fig. 3 shows the results corresponding to the situation where the dipoles form an angle of $\Theta=55^{\circ}$ with the $x$-axis. In this case the dipolar interaction almost vanishes along this direction, favoring the alignment of the particles along it in order to minimize the interaction energy. As in the perpendicular case, one clearly observes the strong enhancement of the interaction effects as the SO coupling is increased, with a 3-peak Wigner-chain structure emerging when $\lambda_{\text {SO }}$ becomes large enough. The generation of SO coupling by means of external laser fields appears thus as a very effective alternative way to increase the effects of the interactions in ultracold dipolar systems. 

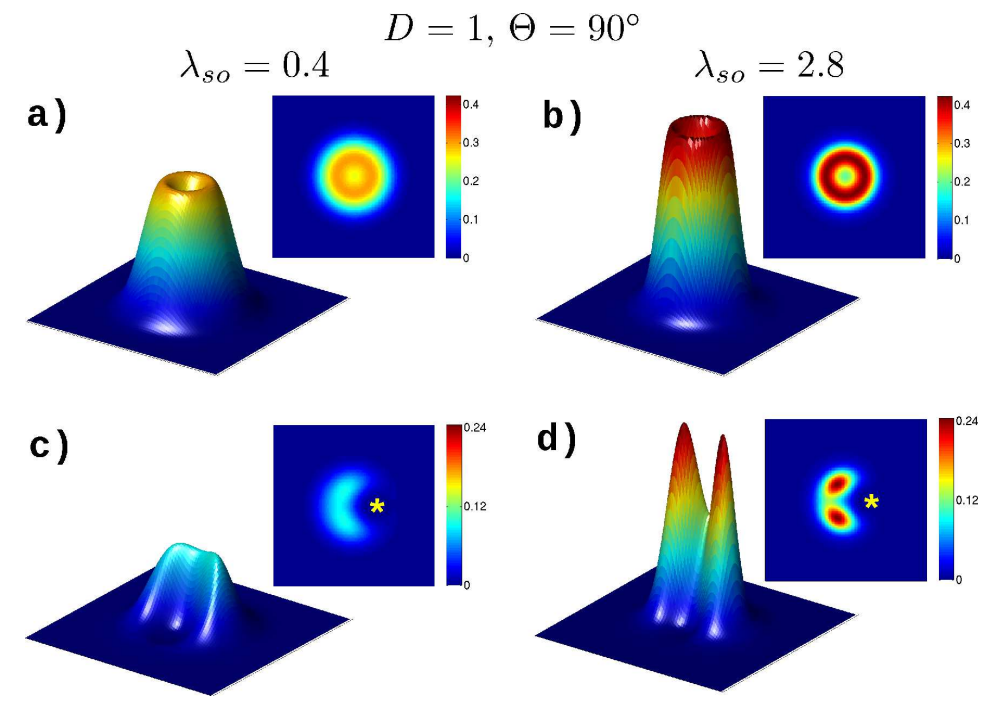

Fig. 1. (Color online) Densities (upper row) and pair-correlated densities (lower row) corresponding to the ground state of the three-boson system with dipole moment perpendicular to the plane of motion $\left(\Theta=90^{\circ}\right)$ and strength $D=1$. The left (a) and c)) and right (b) and d) $)$ columns correspond to weak $\left(\lambda_{S O}=0.4\right)$ and strong $\left(\lambda_{S O}=2.8\right)$ spin-orbit coupling, respectively. Each contour plot, here and in subsequent figures, show the data in the $x y$-plane between the intervals of $-4<x<4$ (horizontally) and $-4<y<4$ (vertically), and the star symbol in the pair-correlated densities refers to the position of the reference particle.



Fig. 2. (Color online) Same as Fig. 1 for $N=4$ and $D=2$.

Finally, we want to mention that the analogy between the repulsively interacting SO-coupled dipolar bosons and their electronic counterparts is not only limited to the Wigner-localization properties. Fig. 4 shows the yrast spectra (i.e., the ground-state energy as a function of the total angular momentum $J_{z}$ ) for three bosons with different strengths of the dipolar interaction and SO coupling. When comparing with the results that were obtained in the electronic case [13, it can be seen that both systems exhibit 


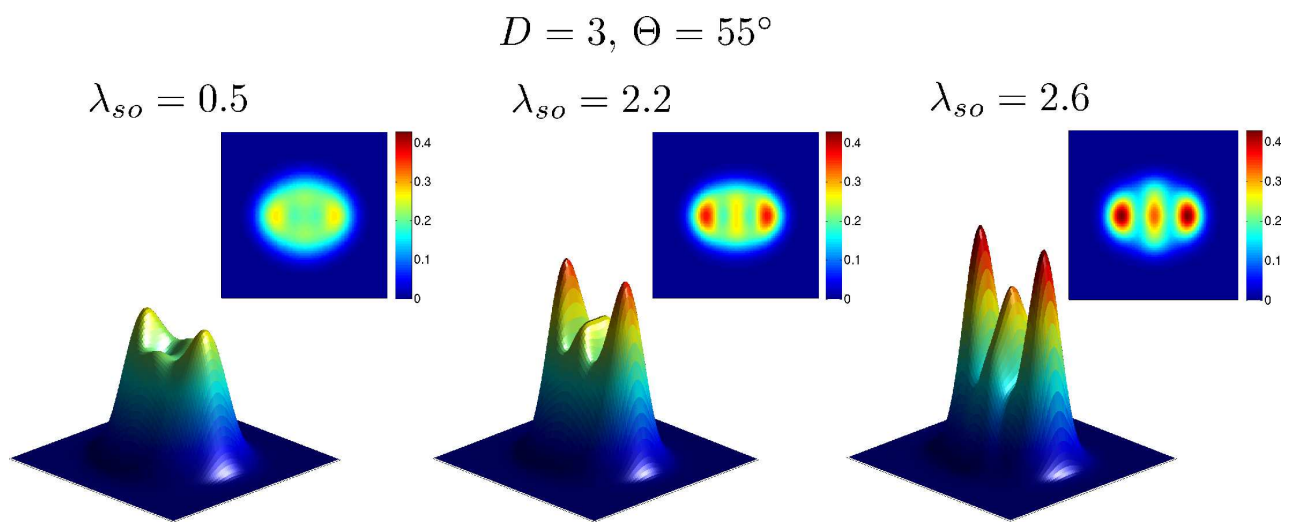

Fig. 3. (Color online) Three tilted dipoles with $\Theta=55^{\circ}, D=3$ and different SO coupling strengths. Each plot has the same color scale, indicated in the figure.

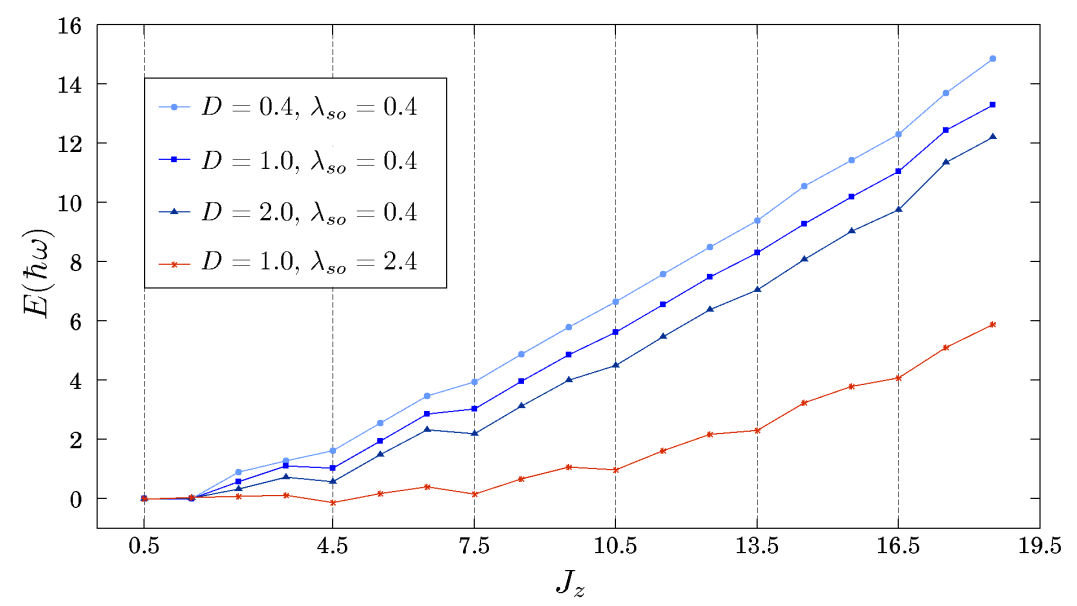

Fig. 4. (Color online) Yrast spectrum of three SO-coupled bosons with isotropic and repulsive dipolar interaction.

nearly a flat spectrum in the low $J_{z}$ range due to the almost degenerate single-particle states in this region. However, as the angular momentum increases, this flat structure evolves into oscillating yrast lines with a period determined by the number of particles in the system. We find that these oscillations become more apparent as the dipoles repel each other more strongly (for the isotropic interaction with $\Theta=90^{\circ}$ ). Moreover, one can see that even when the dipolar interaction is weak the periodic behaviour of the spectrum is preserved due to the action of the SO coupling, illustrating how the latter enhances the effect of the interactions. Such periodic oscillations in the yrast line with recurring local minima are a well-known signature of Wigner localization in electronic systems 1318 . The above results show how bosonic and fermionic systems become similar in the well-localized limit, where the effect of interactions are strong.

\section{Conclusions and outlook}

In this paper we have investigated the interplay between the DDI and the symmetric Rashba SO coupling in a few-body bosonic system confined by a harmonic potential. 
We have shown that the SO coupling strongly enhances the effects of the dipolar interaction, giving rise to Wigner-localized structures whose observation would require much larger dipole strengths in the spin-orbit-free case. This effect is not only observed in the case of isotropic and purely repulsive DDI, where the dipoles are aligned perpendicularly to their plane of motion, but also when they are tilted and the interparticle interaction becomes highly anisotropic with both attractive and repulsive regions.

With a setup for their experimental realization having already been proposed [19], dipolar gases with SO coupling should be expected to allow the observation of novel interesting phenomena due to the non-trivial interplay between the two-body interaction and the Rashba coupling [20, in particular due to the long-ranged and anisotropic nature of the dipole-dipole interaction, as opposed to the more commonly considered contact-type one. The high degree of tunability and controllability of these systems should allow for the observation of the Wigner-localized states reported in this paper.

Finally, we have restricted our discussion here to the Rashba-type SO coupling, which is the most commonly considered one in theoretical studies, but which has not been experimentally realized yet due to its highly symmetric character. The effect of an asymmetric coupling, which may model current experiments in a more realistic way will be investigated in a future work.

Acknowledgements: We thank A. Fetter, G. M. Kavoulakis and A. Cavalli for useful discussions. This work was financially supported by the Swedish Research Council, the Nanometer Structure Consortium at Lund University, and the POLATOM ESF Research network.

\section{References}

1. K. Osterloh et al., Phys. Rev. Lett. 95010403 (2005); J. Ruseckas et al., Phys. Rev. Lett. 95010404 (2005).

2. Y. A. Bychkov and E. I. Rashba, J. Phys. Chem. 176039 (1984); G. Dresselhaus, Phys. Rev. 100580 (1955).

3. Victor Galitski and Ian B. Spielman, Nature 494, 49 (2013).

4. C.Wang et al., Phys. Rev. Lett. 105160403 (2010); Z. F. Xu, R. Lu, and L. You, Phys. Rev. A 83, 053602 (2011); T. Kawakami, T. Mizushima, and K. Machida, Phys. Rev. A 84, 011607 (2011).

5. Y.-J. Lin, K. Jiménez-García, and I. B. Spielman, Nature 47183 (2011).

6. J. Dalibard et al., Rev. Mod. Phys. 831523 (2011).

7. Hui Zhai, Int. J. Mod. Phys. B 26, 1230001 (2012).

8. J.-Y. Zhang et al., arXiv: 1305.7054v1 [cond-mat.quant-gas].

9. Tomoki Ozawa and Gordon Baym, Phys Rev. A 84, 043622 (2011); Xiang-Fa Zhou et al., Phys. Rev. A 84, 063624 (2011); B. Ramachandhran et al., Phys. Rev. A 87, 033627 (2013); Amandine Aftalion and Peter Mason, Phys. Rev. A 88, 023610 (2013).

10. F. Deuretzbacher, J. C. Cremon, and S. M. Reimann Phys. Rev. A 81, 063616 (2010); Sascha Zöllner et al., Phys. Rev. Lett. 107, 035301 (2011); A. S. Arkhipov et al., JETP Lett. 82, 39 (2005).

11. N. Barberan, M. Lewenstein, K. Osterloh, and D. Dagnino, Phys. Rev. A 73, 063623 (2006); K. Osterloh, N. Barberan, and M. Lewenstein, Phys. Rev. Lett. 99, 160403 (2007); M. A. Baranov, H. Fehrmann, and M. Lewenstein, Phys. Rev. Lett. 100, 200402 (2008).

12. S. M. Reimann and M. Manninen, Rev. Mod. Phys. 741283 (2002).

13. A. Cavalli, F. Malet, J. C. Cremon, and S. M. Reimann, Phys. Rev. B 84, 235117 (2011).

14. M. A. Baranov, Phys. Rep. 464, 71 (2008); T. Lahaye et al., Rep. Prog. Phys. 72, 126401 (2009); M. A. Baranov et al., Chem. Rev. 112, 5012 (2012). 
15. G. M. Bruun and E. Taylor, Phys. Rev. Lett. 101, 245301 (2008).

16. J. C. Cremon, G. M. Bruun, and S. M. Reimann, Phys. Rev. Lett. 105255301 (2010).

17. A. G. Volosniev et al., Phys. Rev. Lett. 106, 250401 (2011); A. G. Volosniev et al., Phys. Rev. A 85, 023609 (2012).

18. P. A. Maksym, Phys. Rev. B 53, 10871 (1996).

19. Y. Deng et al., Phys. Rev. Lett. 108, 125301 (2012).

20. Ryan M. Wilson, Brandon M. Anderson, and Charles W. Clark, Phys. Rev. Lett. 111, 185303 (2013). 